\title{
Commentaries
}

\section{The colon and PSC: new liver, new danger?}

The close relation between primary sclerosing cholangitis (PSC) and inflammatory bowel disease (IBD) continues to provide more questions than answers. The prevalence of IBD in patients with PSC is about $55-75 \%$, and PSC occurs in patients with ulcerative colitis at a frequency of $2.5-7.5 \%{ }^{12}$ PSC is found less often in Crohn's disease where it is usually associated with colonic involvement. Recent studies have found an increased prevalence of colonic dysplasia and cancer in patients with PSC and ulcerative colitis compared with ulcerative colitis alone. ${ }^{34}$ The mechanism by which PSC increases the risk of colonic neoplasia is unknown. The increased long term survival of patients with both PSC and ulcerative colitis after liver transplantation may therefore put these patients at an increased risk of developing colorectal cancer in view of the increased neoplastic potential as a result of the immunosuppressive therapy required after liver transplantation. Indeed, some studies have suggested that there may be a higher incidence of colorectal cancer in the first two years after liver transplantation..$^{5-7}$ This may reflect the higher doses of immunosuppression used during this time as after this period, increased rates of colonic cancer have not been reported. However, three studies, including the report by Papatheodoridis et al (see page 639), have been unable to detect higher rates of colonic cancer. ${ }^{8} 9$ Although the data are conflicting, colonic surveillance is mandatory in this high risk group of patients and yearly colonoscopy has been recommended. ${ }^{10}$

Despite the fact that the colitis is usually total in extent it has been a constant feature of all clinical series that the clinical course of the ulcerative colitis is mild or asymptomatic. ${ }^{11}$ It has also been established from retrospective studies that the clinical course and activity of the ulcerative colitis seems to be independent of the natural history and progress of the hepatobiliary disease. ${ }^{12}$ There is evidence from the Mayo Clinic that patients who have had an ileal reservoir created for ulcerative colitis and who also have PSC, have a higher rate of pouchitis. However, the course of pouchitis in PSC is not altered by liver transplantation. ${ }^{12}$

In view of the fact that ulcerative colitis usually has a milder asymptomatic course and that after liver transplantation most patients are treated with immunosuppressive agents, it could be expected that the course of IBD would further improve after orthotopic liver transplantation (OLT). To date, however, the published studies provide contrasting data. ${ }^{13}{ }^{14}$ In the first report from Pittsburgh, 23 patients transplanted for PSC were treated with maintenance immunosuppression with cyclosporin and prednisolone. ${ }^{13}$ All six patients who were asymptomatic before OLT remained well. No patients worsened and 14 $(82 \%)$ of 17 patients with previously active ulcerative colitis reported significant improvement. These results were confirmed at another centre in which remission was maintained with cyclosporin or tacrolimus and corticosteroids with or without the addition of azathioprine or cyclophosphamide. ${ }^{8}$ Eighteen of 27 patients with IBD showed improvement, four patients remained stable and only five had worsening IBD activity after transplantation. ${ }^{14}$ In notable contrast, two other studies have found no difference or worsening in activity after OLT. ${ }^{15}{ }^{16}$ Shaked et al studied 24 patients and found that $33 \%$ worsened after OLT and only $4 \%$ improved. Confirmation of these results came from another recent study where triple immunosuppression was used, ${ }^{17}$ and worsening and progression of IBD symptoms were observed in $31 \%$ of 19 patients. However, it should be noted that the course of post-transplant ulcerative colitis was compared only with the clinical status of colitis in the immediate pretransplant period.

Papatheodoridis et al have reported the results from the Royal Free Hospital, a transplant unit in which corticosteroids are withdrawn three months after transplantation and patients are then maintained with either cyclosporin or tacrolimus with or without azathioprine. They have described 30 patients who were transplanted for PSC and survived more than 12 months. The clinical course of ulcerative colitis after OLT compared with that up to five years before transplantation was the same in $50 \%$ and worse in $50 \%$. Two thirds of patients who were quiescent beforehand remained quiescent and one third worsened, whereas all patients who had had an active pretransplant course developed significant worsening after liver transplantation. Also of interest, despite immunosuppression, three of 12 patients with PSC, without previous evidence of IBD, developed active colitis after transplantation. However, exacerbation of colitis occurred only after steroid withdrawal.

What do these results mean? Current evidence suggests that after OLT ulcerative colitis may pursue a more aggressive course or even develop for the first time in patients in whom corticosteroids are withdrawn and where other immunosuppressive drugs are used. There is no obvious explanation for these surprising findings. Papatheodoridis and coworkers suggest that the presence of normally functioning liver creates a new balance in immunoregulation, pointing out that patients with advanced liver disease often have depressed $\mathrm{T}$ cell function as well as other immune system disturbances. An alternative explanation may be that altered bile salt concentrations after OLT may adversely affect colonic function.

To date, the aetiology and pathogenesis of PSC and its association with IBD remain unknown. Cholangiocytes and colonocytes may share common autoantigens or bacterial products such as formyl peptides which may ascend the portal vein to excite hepatobiliary change in susceptible patients. Further insights into the disease mechanisms involved in the pathogenesis of IBD may elucidate the paradox of the mild clinical course of total colitis before liver transplantation with significant worsening after transplantation.

Fohn Radcliffe Hospital,

R W CHAPMAN

Headington, Oxford OX3 9DU, UK

1 Chapman RWG, Arborgh BAM, Rhodes JM, et al. Primary sclerosing cholangitis: a review of its clinical features, cholangiography, and hepatic histology. Gut 1980;21:870-7.

2 Balan V, LaRusso NF. Hepatobiliary disease in inflammatory bowel disease. Gastroenterol Clin North Am 1995;24:647-69. 
3 Broome U, Lindberg G, Lofberg R. Primary sclerosing cholangitis - a risk factor for the development of dysplasia and DNA aneuploidy. Gastroenter(10)

4 Gurburz AK, Giardiello FM, Bayless TM. Colorectal neoplasia in patients with ulcerative colitis and primary sclerosing cholangitis. Dis Colon Rectum 1995;38:37-41.

5 Higashi H, Yanaga K, Marsh JW, et al. Development of colon cancer after liver transplantation for primary sclerosing cholangitis associated with ulcerative colitis. Hepatology 1990;11:477-80.

6 Bleday R, Lee E, Jessurum J, et al. Increased risk of early colorectal neoplasms after hepatic transplant in patients with inflammatory bowel disease. Dis Colon Rectum 1993;36:908-12.

7 Knechtle SJ, D'Alessandro AM, Harms BA, et al. Relationships between sclerosing cholangitis, inflammatory bowel disease, and cancer in patients undergoing liver transplantation. Surgery 1995;118:615-20.

8 Shaked A, Colonna JO, Goldstein L, et al. The interrelation between sclerosing cholangitis and ulcerative colitis in patients undergoing liver transplantation. Ann Surg 1992;215:598-605.

9 Marotta PJ, Graziadei IW, Wiesner RH, et al. Inflammatory bowel disease in patients with primary sclerosing cholangitis receiving liver transplantation patients with primary sclerosing cholangitis
[abstract]. Hepatology 1997;26(suppl):237A.

10 Loftus EV, Aguilar HI, Sandborn WJ, et al. Risk of colorectal neoplasia in patients with primary sclerosing cholangitis and ulcerative colitis following orthotopic liver transplantation. Hepatology 1998;27:686-9.
11 Lundqvist K, Broome U. Differences in colonic disease activity in patients with ulcerative colitis with and without primary sclerosing cholangitis: a case control study. Dis Colon Rectum 1997;40:451-6.

12 Zins BJ, Sandborn WJ, Penna CR, et al. Pouchitis disease after orthotopic liver transplantation in patients with primary sclerosing cholangitis and an ileal pouch-anal anastomosis. Am f Gastroenterol 1995;90:2177-81.

13 Galvaler JS, Delemos B, Belle SH, et al. Ulcerative colitis disease activity as subjectively assessed by patient-completed questionnaires following orthotopic liver transplantation for sclerosing cholangitis. Dig Dis Sci 1991;36:321-8.

14 Kitis G, Wright Hl, Abu-Elmagad K, et al. The course of inflammatory bowel disease under FK 506 immunosuppression after liver transplantation for primary sclerosing cholangitis [abstract]. Heptology 1992;16(suppl): $191 \mathrm{~A}$

15 Stephens J, Goldstein R, Crippin J, et al. Effects of orthotopic liver transplantation and immunosuppression on inflammatory bowel disease in primary sclerosing cholangitis patients. Transplant Proc 1993;25:1122-3.

16 Fabry TL, Klion FM, Post JA, et al. Outcome of liver transplantation (OLTX) for primary sclerosing cholangitis (PSC) and the effect of immunosuppression on the course of inflammatory bowel disease [abstract]. Hepatology 1993;18(suppl):69A.

17 Narumi S, Roberts JP, Emond JC, et al. Liver transplantation for sclerosing cholangitis. Hepatology 1995;22:451-7.

\section{Multiple HNPCC tumours: ask the family!}

Hereditary non-polyposis colorectal cancer (HNPCC) is one of the most common conditions predisposing to colorectal cancer (CRC). Affecting less than $1 \%$ of the general population, HNPCC confers a lifetime risk of CRC estimated at over $80 \%$; the risk to age 40 may exceed $30 \%{ }^{1}$ It can be caused by inherited mutations in one of the several DNA mismatch repair (MMR) genes. The resulting DNA MMR deficiency in neoplastic tissue gives rise to microsatellite alterations, a reflection of genomic instability. ${ }^{2}$ Patients with HNPCC are at increased risk of cancers other than CRC, including cancers of the endometrium, ovary, stomach, small bowel, and the upper urinary tract (renal pelvis and ureter). ${ }^{3}$

Despite recent advances in the understanding of the molecular genetic basis of HNPCC, we are still far from the clinical ideal of being able to identify patients with this disorder among the population we serve. Tests for mutations in MMR genes are problematic. There are classic HNPCC families with clear linkage to one of the MMR genes which have been exhaustively but fruitlessly searched for mutations, indicating some types of HNPCC associated mutations are not identifiable by current technologies. Even when an inherited alteration of an MMR gene is discovered, its causal role can be difficult to establish. These and other problems thwart the application of mutation testing in the general population.

Conversely, in families with good evidence of HNPCC, the clinical importance of DNA testing is widely accepted. Initial discovery of a disease associated inherited MMR mutation in an affected family member can be very costly, but it makes possible the testing of unaffected, high risk family members for this specific mutation at greatly reduced cost. Prior to testing, all the high risk unaffected family members are regarded as having high cancer risk. Consequently, they must consider various onerous and expensive alternatives including intensive screening and prophylactic surgery. Testing frees those without the mutation from this burden, and may help those with the mutation to cope with it because their risk is less uncertain.

The clinical importance of simply recognising an HNPCC family is widely accepted, even in the absence of genetic testing. Based on the autosomal dominant mode of inheritance and the cancer diagnoses in the family, an individual's risk of carrying the putative mutation can be calculated and genetic counselling with cancer prevention methods recommended. The sparse evidence available indicates that CRC diagnoses and deaths were reduced in subjects undergoing colonoscopy screening. ${ }^{4}$

In this situation the clinician's most important role is to recognise patients who should be referred to genetics centre for evaluation. Brown et al, in this issue (see page 664), provide strong empirical evidence that a patient with multiple HNPCC spectrum tumours, or with a close relative with such tumours, should be investigated in this way. They compared the family history of cancer in two groups of patients: those with CRC and another HNPCC associated primary cancer (colorectal, gastric, urinary, ovary, uterine), and those with a single primary CRC. Thirteen per cent of the family histories from the multiple primary cancer group were suggestive of HNPCC, compared with less than $1 \%$ from the single primary CRC group. These findings reinforce the importance of obtaining comprehensive family histories of cancer of all anatomical sites in patients with CRC, and stress the importance of multiple primary HNPCC associated cancers as a "red flag" to the diagnostician. Identifying HNPCC patients/families enables atrisk relatives to benefit from targeted surveillance and management programmes which are melded to HNPCC's natural history.

The authors' findings should not surprise those who have studied hereditary cancer. Many of these disorders show specific patterns of multiple primary cancers. Another form of hereditary CRC provides a classic example: in familial adenomatous polyposis (FAP), polyposis or CRC can occur with periampullary carcinoma, gastric carcinoma, papillary thyroid carcinoma, brain tumours, carcinoma of the small bowel, carcinoma of the pancreas, and desmoid tumours. An alert physician will recognise that a patient whose parent had CRC and desmoid tumours should be investigated for FAP; the combination of tumours provides the clue.

What is the importance of the specific pattern of multiple primary cancer associated with HNPCC? Brown et al have provided a clear message to the diagnostician: take a careful family history and be aware of the tumour spectrum in HNPCC! The importance for the basic scientist remains elusive, but clearly the tumour spectrum itself provides a clue to the effect of the MMR genes. The several affected 
tissues undergo malignant transformation at variable rates. The colon is the commonest cancer site, followed by endometrium. The remaining sites are much less common. The reason for the primacy of CRC is unclear, but it may be related to environmental factors. Old records of HNPCC families in the USA indicate that gastric cancer was once commoner than CRC. ${ }^{78}$ Today, HNPCC families from Japan show more gastric cancer than is typical in western HNPCC families. ${ }^{9}$ Fearon touches on the most likely explanation in his review of human cancer syndromes ${ }^{10}$ : the sites at risk must be exposed to an environmental injury that makes mutation or inactivation of the wild type allele more likely there.

Why does HNPCC affect these tissues and not others? Why, for example, do the ureter and renal pelvis show higher rates of cancer than the urinary bladder, given that all are lined with transitional cells which are morphologically the same? There are fewer cells in the ureter than in the bladder, and exposure to carcinogens in urine is briefer. Environmentally induced urinary tract cancer is usually observed in the bladder. In aniline dye workers, occupational exposure gives rise to urinary bladder cancer much more frequently than ureteral and renal pelvis cancer. We know of no data that can explain the particular pattern of sites of cancer occurrence in HNPCC.

Despite this underlying biological mystery, we know which extracolonic sites are at high risk for malignancy in HNPCC and Brown et al reinforce the clinical importance of this information. The first step in HNPCC cancer prevention is recognition of the syndrome and so clinicians must investigate the cancer family history, including all anatomical sites and know the cardinal features of the syndrome, including the pattern of multiple primary cancers.

H T LYNCH

P WATSON

Department of Preventive Medicine,

Creighton University School of Medicine,

2500 California Plaza,

Omaha, Nebraska 68178, USA

1 Vasen HFA, Wijnen JT, Menko FH, et al. Cancer risk in families with hereditary nonpolyposis colorectal cancer diagnosed by mutation analysis. Gastroenterology 1996;110:1020-7.

2 Kinzler KW, Vogelstein B. Lessons from hereditary colon cancer. Cell 1996; 87:159-70.

3 Watson P, Lynch HT. Extracolonic cancer in hereditary nonpolyposis colorectal cancer. Cancer 1993;71:677-85.

4 Järvinen HJ, Mecklin J-P, Sistonen P. Screening reduces colorectal cancer Järvinen HJ, Mecklin J-P, Sistonen P. Screening reduces colorectal cancer
rate in families with hereditary nonpolyposis colorectal cancer. Gastroenterrate in families with heredita
ology 1995;108:1405-11.

5 Vasen HFA, Taal BG, Nagengast FM, et al. Hereditary nonpolyposis colorectal cancer: results of long-term surveillance in 50 families. Eur $\mathcal{F}$ Cancer 1995;31A: 1145-8.

6 Lynch HT, Smyrk T. Hereditary nonpolyposis colorectal cancer (Lynch syndrome): an updated review. Cancer 1996;78:1149-67.

Warthin AS. Heredity with reference to carcinoma. Arch Intern Med 1913;12:546-55.

8 Lynch HT, Krush AJ. Cancer family "G” revisited: 1895-1970. Cancer 1971;27:1505-11.

9 Sakiyama T, Sakanoue Y, Miki Y, et al. An attempt for identification of hereditary nonpolyposis colorectal cancer in Japan. In: Utsunomiya J, Lynch HT, eds. Hereditary colorectal cancer. Tokyo: Springer-Verlag, 1990:219-24.

10 Fearon ER. Human cancer syndromes: clues to the origin and nature of cancer. Science 1997;278:1043-50.

\section{MRCP: examining the obstructed bile duct}

Clinicians have been taken aback by the rapid developments in magnetic resonance cholangiopancreatography (MRCP), including elegant computer reconstructions of the biliary and pancreatic ducts from images acquired by magnetic resonance scanning. Pioneer endoscopists of the late 1970 s and 1980 s struggled to produce diagnostic retrograde cholangiograms, at some risk to the patient, and now in the 1990s images of almost comparable quality can be produced without an endoscope, without contrast and even without radiation. The technical aspects are so complex that the non-radiologist is unlikely to understand the variations such as T2 spin weighting, half-Fourier acquisition single-shot turbo spin-echo (HASTE) sequences, and rapid acquisition with relaxation enhancement (RARE). For those wishing to try, they are clearly covered in a recent leading article in this journal. ${ }^{1}$ Yet the technique is so beguiling and the images so similar to those obtained with contrast injection that clinicians will have to be wary of serious errors of misinterpretation, particularly when viewing an image in just one plane or with one technique. For instance, there are artefacts produced by flow and by adjacent structures such as vessels that can mimic luminal filling defects, and some beautifully illustrated examples have been published recently. ${ }^{2}$ Also, it should be emphasised that many clinicians will be disappointed when they demand such images from their radiology department. Technology is moving so fast that only the most recent and versatile machines are likely to have the facilities available for high quality MRCP.

Already there are reports of its use in every conceivable condition, from carcinoids ${ }^{3}$ to cholangiomas. ${ }^{4}$ There are also many direct comparisons between MRCP and endoscopic retrograde cholangiopancreatography (ERCP) where the newer technique produces sensitivities and specificities above $70 \%{ }^{5}$ or even $90 \%,{ }^{6}$ but the evidence of how and when MRCP should be used is emerging more slowly. The study by Adamek et al in this issue (see page 680) goes some way to defining this in the common clinical scenario of suspected bile duct obstruction. Clearly, one aim should be to avoid unnecessary ERCPs and in this context their results and those of others ${ }^{7}$ show a high negative predictive value-a normal MRC makes bile duct pathology unlikely. Unfortunately, in Adamek et al's series there were only three patients in 60 who turned out to have ductal calculi, and we know that in clinical practice these are the most problematic ones because of the difficulty in imaging the duct by ultrasonography ${ }^{8}$ and the observation that the biliary system may well not be dilated-in distinction to malignant obstruction. Every endoscopist will tell you that stones may emerge after a sphincterotomy undertaken because of a high index of clinical suspicion in the face of a normal, high quality retrograde cholangiogram.

MRCP may be non-invasive and completely safe, but it is an expensive use of a valuable resource. Before we reach for the "MR request form" we should make sure we are already using existing evidence to exclude biliary disease by a combination of careful ultrasonography and biochemistry. ${ }^{9}$ We should not be undertaking ERCP now in patients with non-specific abdominal pain, normal liver function tests and normal ultrasonography, and so we should not be substituting MRCP either. At the other end 
of the spectrum, there is no point in doing MRCP in patients with unequivocal biochemical and ultrasonographic evidence of extrahepatic cholestasis-for example, the typical elderly patient with jaundice and dilated intrahepatic bile ducts. Such patients are going to benefit from the therapeutic potential of the endoscopic approach and no benefit is likely to accrue from costly duplication. Most surgeons like endoscopic stenting and biliary drainage as a preliminary to resection of pancreatic and bile duct tumours and unless this changes, ERCP will remain the method of choice for preoperative cholangiography. The quality of magnetic resonance is likely to continue to improve, and it may have a future role as a single, "one-stop" assessment of operability in such patients because of the potential to show blood vessels elegantly, as well as other structures. ${ }^{10}$ An area where MRCP has already shown itself to be invaluable in clinical decision making is the patient where endoscopic access to the bile duct is impossible, particularly because of previous surgery. ${ }^{11}$

The report from Adamek et al, together with at least 15 other publications in the first quarter of this year, helps to define the current position of MRCP. The algorithm for investigating suspected bile duct pathology, even for those clinicians fortunate to have the full range of high quality imaging and ERCP available, still starts with biochemical liver function tests and ultrasonography. Where both are normal, further biliary investigations are rarely justified. If there is clear evidence of extrahepatic obstruction and likely therapeutic potential, ERCP is the next investigation of choice, but for those in the equivocal group or where ERCP has failed, MRCP may provide a very useful alternative.

Royal Liverpool University Hospital,

I T GILMORE

Prescot Street, Liverpool L7 8XP, UK

1 Bearcroft PW, Lomas DJ. Magnetic resonance cholangiopancreatography. Gut 1997;41:135-7.

2 David V, Reinhold C, Hochman M, et al. Pitfalls in the interpretation of MR cholangiopancreatography. AfR Am f Roentgenol 1998;170:1055-9.

3 Gembala RB, Arsuaga JE, Friedman AC, et al. Carcinoid of the intrahepatic ducts. Abdom Imaging 1993;18:242-4.

4 Kaneko K, Honda H, Kajiyama K, et al. Radiologically identifiable intratumoral portal vein in intrahepatic cholangiomas: a diagnostic pitfall. Abdom Imaging 1996;21:445-7.

5 Hintze RE, Adler A, Veltzke W, et al. Clinical significance of magnetic resonance cholangiopancreatography (MRCP) compared to endoscopic retronance cholangiopancreatography (MRCP) compared to endoscopic re
grade cholangiopancreatography (ERCP). Endoscopy 1997;29:182-7.

6 Chan YL, Chan AC, Lam WW, et al. Choledocholithiasis: comparison of MR cholangiography and endoscopic retrograde cholangiography. Radiology 1996;200:85-9.

7 Soto JA, Barish MA, Yucel EK, et al. Magnetic resonance cholangiography: comparison with endoscopic retrograde cholangiopancreatography. Gastroenterology 1996;110:589-97.

8 Tobin MV, Mendelson RM, Lamb GH, et al. Ultrasound diagnosis of bile duct calculi. BMF 1986;293:16-17.

9 Thornton JR, Lobo AJ, Lintott DJ, et al. Value of ultrasound and liver function tests in determining the need for endoscopic retrograde cholangiopancreatography in unexplained abdominal pain. Gut 1992;33:1559-61.

10 Govil S, Govil S, Justus A, et al. Magnetic resonance cholangiography as sole pre-operative determinant of resectability in hilar cholangiocarcinoma. Indian 7 Gastroenterol 1997;16:103-4.

11 Adamek HE, Weitz M, Breer $\mathrm{H}$, et al. Value of magnetic-resonance cholangio-pancreatography (MRCP) after unsuccessful endoscopicretrograde cholangio-pancreatography (ERCP). Endoscopy 1997;29:741-4. 